\title{
Avant-propos au rapport géologique Malpasset de Jean Goguel (1960)
}

B. GOGUEL

Coyne et Bellier 9 , allée des Barbaniers 92632 Gennevilliers Cedex bgl@coyne-et-bellier.fr
Trois jours après la rupture catastrophique, le 2 décembre 1959, du barrage de Malpasset, le ministre de l'Agriculture créait une commission ${ }^{1}$ chargée d'enquêter sur les causes de la rupture. Cette Commission « s'est assuré le concours de M. Jean Goguel, ingénieur général des Mines », dont le rapport géologique daté du 17 avril 1960, Annexe 38 à son rapport, n'a eu qu'une diffusion restreinte. La Revue française de géotechnique a souhaité y remédier.

Ce document doit être situé dans le contexte : il est antérieur aux conclusions des travaux et confrontations des deux collèges d'experts ${ }^{2}$ et contre-experts ${ }^{3}$ désignés par le juge d'instruction de Draguignan, qui ont déposé leurs rapports le 10 février 1961 (avec un complément le 10 janvier 1962) et le 7 août 1963, avant de s'en expliquer lors des audiences d'octobre 1964 et d'octobre 1965 du tribunal de grande instance de Draguignan.

La revue Le Génie Civil a publié le rapport provisoire de la commission administrative le $1^{\mathrm{er}}$ juin 1960, puis l'essentiel des rapports des experts judiciaires (1 $1^{\mathrm{er}} \mathrm{et}$ 15 juin 1965 pour celui du premier collège, $1^{\text {er }}$ janvier et mars 1966 pour celui des contre-experts).

Jean Goguel (1908-1987) était à l'époque directeur du service de la Carte géologique de la France, et directeur adjoint du Bureau de recherches géologiques et géophysiques (BRGG), ancêtre du BRGM qui a fusionné les deux organismes en 1968. Professeur à l'École des mines de Paris de 1933 à 1983, de géologie appliquée à I'Ecole des ponts et chaussées de 1941 à 1955 et à l'École du génie rural de 1951 à 1958, il publiait un livre résumant son expérience à cet égard: Application de la géologie aux travaux de l'ingénieur (Masson 1959, 357 p., 2éd. 1967, 370 p.). Il fut le premier président du Comité français de géologie de l'ingénieur.

Dans un texte de 19814, Jean Goguel évoque ainsi sa participation à la commission d'enquête administrative : “ Après l'accident de Malpasset, il était évident qu'une grande faille à remplissage argileux avait permis le glissement du coin rocheux sur lequel reposaient les fondations. La seule question qui se posait était : aurait-il été possible d'identifier ce risque à l'avance et pourquoi cela n'avait-il pas été fait? Lorsque le président du tribunal de Draguignan, après avoir entendu les 18 experts, en a fait ranger un certain nombre à droite, et d'autres à gauche ${ }^{5}$, je me suis retrouvé seul au milieu et, invité à préciser ma position, j'ai dit "les études géologiques ont été insuffisantes, mais je ne suis pas sûr qu'elles auraient permis de reconnaître cette faille". Il est trop facile, après coup, d'affirmer une suffisance technologique, qui n'est pas toujours fondée.

« Par la suite, dans un texte publié par Le Monde 6 , j'ai mis en évidence une autre responsabilité. L'avant-projet du barrage de Malpasset avait été étudié par l'administration responsable sur ses crédits de fonctionnement, donc très sommairement. Et la loi de budget [...] a ouvert la totalité du crédit de construction, pour ce site, spécifié. Cela ne permettait pas, en une époque où la monnaie perdait $10 \%$ de sa valeur chaque année, de consacrer aux études les années qui auraient été nécessaires. Alors que l'EDF a de nombreux dossiers en cours d'étude, et peut choisir ceux qu'elle fait passer dans chaque tranche d'investissement, à Malpasset les études devaient être poussées trop vite, et leur conclusion ne pouvait être que

\footnotetext{
(1) Commission administrative : MM. Gosselin, Ollivier Martin, Calvet, Disserens, Duffaut, Talureau. (2) Premier collège d'experts judiciaires : MM. Casteras, Escande, Gridel, Haegelen, Jacobson, Roubault.

(3) Contre-experts judiciaires : MM. Barbier, Biarez, Caquot, Drouhin, Mary, Pruvost.

(4) Notice Jean Goguel, Travaux dans le domaine de la géologie appliquée, septembre 1981

(5) Confrontation des experts, au matin du samedi 24 octobre 1964 (sténo VII-31), auxquels le président demande de se ranger en partisans de l'imprévisibilité, et partisans de la prévisibilité (qui restent, minoritaires, au nombre de 4).

(6) “ La lumière et la justice », journal Le Monde, 13 mars 1965, page 14. 11 avait déjà formulé cette idée à l'audience (22/10/1964, sténo III-14).
} 
le passage à la construction, ou l'aveu (qui n'aurait pas été justifié) d'une impossibilité. Mais une telle responsabilité ne peut être mise en cause. »)

Dans le même texte ( la lumière et la justice », Jean Goguel déplore que la mort d'André Coyne (survenue le 21 juillet 1960, des suites d'un cancer) ait empêché son inculpation, et donc l'examen détaillé des conditions dans lesquelles il avait travaillé et les facteurs l'ayant induit en erreur. Il souligne que les experts entendus par le tribunal appartenaient à trois commissions qui ont travaillé d'une manière très indépendante, et écrit :

“ Si les spécialistes d’une même discipline, appartenant aux différentes commissions, avaient pu discuter ensemble, un accord se serait sans doute réalisé sur beaucoup plus de points qu'il n'est apparu lors de leur confrontation. En particulier, l'avis de la deuxième commission judiciaire concluant au caractère imprévisible de la catastrophe avec les connaissances de l'époque [...] est fondé sur une théorie nouvelle - celle des sous-pressions agissant à grande profondeur - qui a été conçue [...] après que la commission administrative et la première commission judiciaire eurent terminé leurs travaux. Ces commissions n'ont donc pas pu l'examiner, et leurs membres ont dû se faire individuellement leur opinion sur ce point essentiel, le plus souvent sans bien connaître les discussions auxquelles cette théorie pouvait donner lieu. Une discussion approfondie, contradictoire et critique, par l'ensemble des experts, aurait été nécessaire ; elle était impossible au palais de justice de Draguignan, où nous ne pouvions apporter que des conclusions débarrassées de leur appareil technique. »

L'analyse de la rupture et sa discussion technique ont été développées par la suite comme en rendent compte d'autres contributions à ce même numéro de la Revue (P. Duffaut et P. Habib, A. Carrère).

Pour mémoire et info du comité de rédaction de la Revue Française de géotechnique, voici ce qu'il avait déclaré alors :

" Malheureusement, lorsque la décision de construction a été prise et que les crédits nécessaires ont été attribués par le ministère de l'Agriculture au département, la subvention a été accordée pour la construction. Il ne faut pas oublier qu'on était dans une période oì le franc perdait $10 \%$ de sa valeur chaque année. Par conséquent, le maître de l'œuvre avait le souci d'aller aussi vite que possible pour pousser sa construction lorsque les crédits lui permettaient encore de la faire. Il n'est pas douteux que s'il avait agi autrement, on aurait pu l'accuser d'impéritie. Il est très regrettable que les crédits n'aient pas été accordés en deux phases: d'abord des crédits d'études larges, permettant l'établissement d'un avant-projet détaillé et comportant tout ce qu'il fallait savoir pour l'édification de ce barrage et, des années plus tard, les crédits pour la construction proprement dite de l'ouvrage. )

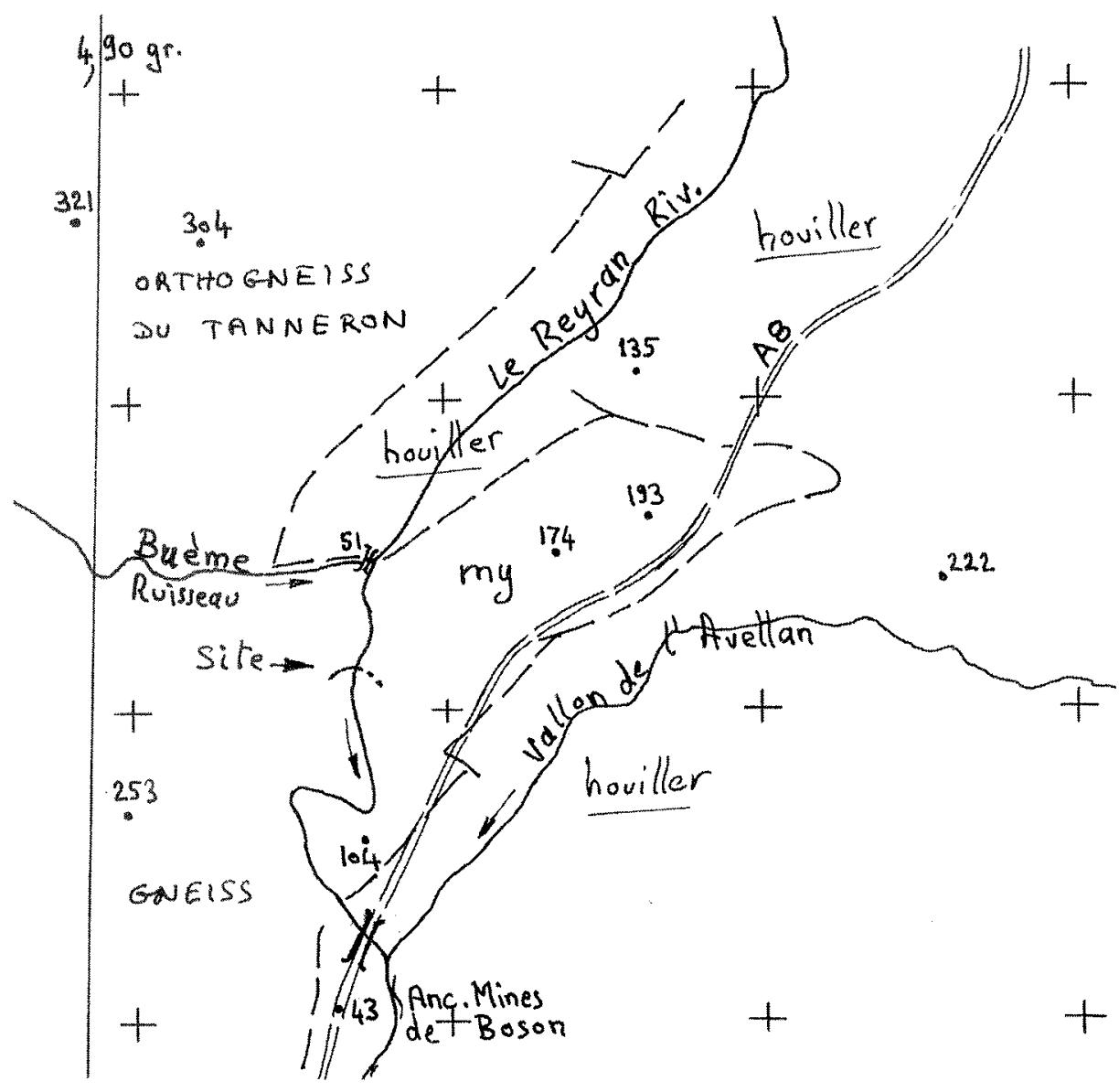

Cadre géologique du site de Malpasset

Carroyage kilométrique UTM-WGS84 et points cotés selon carte IGN TOP25 n 3544 ET.

Limite Gneiss / Houiller en tiretés selon carte géologique de la France à 1/50 000, feuille Fréjus-Cannes, 2e édition, 1994 my: gneiss mylonitiques œillés.

(Document préparé par Bernard Goguel, sous l'amicale pression de Pierre Duffaut). 